\title{
A descriptive clinical evaluation of arthroscopic synovectomy in rheumatoid knees: a prospective study
}

\author{
Sharath K Rao', Sripathi Rao ${ }^{2}$ and A Monappa Naik ${ }^{3}$ \\ (Index words: Function, pain, perioperative morbidity, range of motion)
}

\begin{abstract}
Objective To evaluate the clinical outcome of arthroscopic synovectomy for persistent rheumatoid synovitis of the knee joints.

Design Prospective clinical study.

Setting Kasturba Medical College Hospital, Manipal.

Patients Fifty two knee joints in 46 patients.

Measurements The effect of the procedure and its influence in the progression of the disease process on knee joints were assessed in terms of reduction of pain, improvement in range of motion, improvement in functional activity and recurrence of synovitis with effusion.
\end{abstract}

Results During the average follow up period of 5 years, the patients showed appreciable improvement $(90 \%$ of knee joints) until 3 years of follow up. At the end of 5 years of follow up, it reduced to about $75 \%$.

Conclusions Arthroscopic synovectomy along with medical treatment can control the disease process and preserve the knee joint function for up to 3 years.

\section{Introduction}

Descriptions of the surgical excision of the synovium from a variety of joints have been published for over a century. Open synovectomy of the knee, used first for the treatment of tubercular arthritis, was later used in rheumatoid arthritis. The main disadvantage of open synovectomy of the knee is related to the rehabilitation of the patient after the surgical procedure. Most authors recommended a need for manipulation of the knee under anaesthesia during the early post-operative period to overcome loss of motion. Extensive physical therapy and prolonged hospital stay are often necessary to obtain a good result [1]. Wound separation, haematoma, infection and cutaneous nerve injury represent other documented complications.

The enlargement of the field of application of arthroscopy from diagnostic use to therapeutic purposes and the development of new instruments such as the motorised intra-articular shaver have facilitated safe and efficient arthroscopic synovectomy. The main change is in the technique rather than the concept or indications.
Our study was designed to clinically evaluate the efficacy, tolerance, and morbidity of arthroscopic synovectomy in a group of patients with persistent active rheumatoid synovitis, by comparison of pre-operative and postoperative pain, range of movement and functional activity, and recurrent synovitis with effusion.

\section{Materials and methods}

This is a prospective study of 46 patients ( 52 knees) of arthroscopic synovectomy of the knee done in Kasturba Medical College Hospital, Manipal, India, from December 1997 to January 2000. Rheumatoid arthritis patients with persistent synovitis in the joint causing pain and swelling despite 6 months of adequate medical management were included in the study. All the patients had loss of functional activity and a reasonable range of motion. Patients with marked destruction and deformity of the joint, and those who had previously undergone open synovectomy were excluded from the study.

After clinical examination and relevant investigations, the diagnosis of rheumatoid arthritis was established. The patients were scored pre-operatively using the criteria of pain, range of motion, functional activity and synovitis with effusion. All operations were done under general or spinal anaesthesia with a pneumatic tourniquet. Synovectomy was performed in both anterior and posterior compartments using four primary portals, namely, superolateral, superomedial, inferolateral and inferomedial. If necessary, additional portals were made to gain access to the posterior compartment. The basic instrument used consisted of a $5.5 \mathrm{~mm}$ full radius synovial resector, which was used in oscillating mode. Pressure irrigation was used for distension of the joint throughout the procedure using a gravity flow system.

Post-operatively, a compression bandage was applied leaving one or two portals open for drainage. The compression dressings were removed on the seventh postoperative day and progressive weight bearing started. The patients were placed on an exercise regimen to strengthen the quadriceps and hamstring muscles and to regain the range of motion. Standardised medical treatment was

${ }^{1,2}$ Professors and ${ }^{3}$ Assistant Professor, Department of Orthopaedics, Kasturba Medical College, Manipal, Karnataka 576104, India.

Correspondence: SKR, Tel. +919845177418, e-mail: <skraok@yahoo.com>. (Competing interests: none declared). Received 22 September 2005 and revised version accepted 11 November 2005. 
Table 1. Number of knee joints with clinical criterion at each follow up $(\mathrm{N}=52)$

\begin{tabular}{lrrrrrrrr}
\hline Months of follow up & 1 & 3 & 6 & 12 & 24 & 36 & 48 & 60 \\
\hline Improvement in pain & 46 & 50 & 50 & 50 & 50 & 47 & 44 & 41 \\
Improvement in ROM & 52 & 50 & 50 & 50 & 47 & 45 & 42 & 39 \\
Improvement in function & 40 & 52 & 52 & 50 & 50 & 47 & 43 & 37 \\
No recurrence of synovitis & 42 & 52 & 52 & 50 & 50 & 42 & 37 & 28 \\
\hline
\end{tabular}

$\mathrm{ROM}=$ range of motion

maintained throughout.

Patients were reviewed at 1, 3 and 6 months and after that every year. At each visit patients were scored for pain, range of motion, functional activity, and recurrent synovitis with effusion. The scores obtained under each criterion were evaluated using the modified score developed for evaluation of knee synovectomy, originally proposed in 1974 by Laurin [1].

\section{Results and analysis}

There were 46 patients, and six patients had bilateral involvement, making 52 knees, 26 of them were males. The ages ranged from 11 to 63 years (mean age 30.3 ). The minimum duration of pain was for 6 months and the maximum 60 (mean age 23.5). All 46 patients had pain and swelling, and $34(74 \%)$ had reduced range of movement. The average hospital stay after operation was 1.8 days with a range of $1-8$ days (Table 1 ).

\section{Pain}

During follow up, until the second year, an improvement in pain (from $88 \%$ to $96 \%$ ) was noted, which was not statistically significant $(p=0.14)$. There was a significant worsening of pain in the following 3 years (from $96 \%$ to $78 \%$ ), with $\mathrm{p}$ value of 0.01 . But over a total 5 years of follow up, no significant worsening of pain (from $88.5 \%$ to $78 \% ; \mathrm{p}=0.19$ ) occurred.

\section{Range of motion (ROM)}

No significant loss in ROM in the first 2 years was noted. Over a period of 3 years there was a significant loss in ROM ( $p=0.01)$, which worsened in the following years.

\section{Functional activity}

There was a significant loss of function 1 month after surgery $(p=0.001)$, which was completely regained in the next 2 months. At the end of 3 years there was a significant loss of function (from $100 \%$ to $90 \%)(p=0.02$ ) which correlates with worsening of pain and loss of ROM.

\section{Synovitis and effusion}

Eighty per cent of the patients showed improvement in achieving good or excellent results at the end of 1 month. The improvement increased to $100 \%$ at the end of 3 and 6 months, and then reduced to $96 \%$ at the end of 12 months and 2 years. The relatively small improvement at the end of 1 month was due to recurrent effusion postoperatively, which gradually settled at the end of 3 months. Recurrent synovitis was frequently noted during further follow up.

\section{Discussion}

Arthroscopic synovectomy has been developed as an effective tool for the ablation of the synovium in the knee joint. The procedure must be done in good time (early synovectomy) and be both radical and tissue sparing [2]. Effective and safe methods of access to different compartments of the knee have been developed. Providing that proper precautions be taken, the procedure can be carried out safely and with minimum morbidity [3]. In our study, $96 \%$ of the operated knees showed good results at the end of 6 months, that was maintained at the end of 12 months. This result gradually reduced to $90 \%$ and to $80 \%$ at the end of 4 and 5 years of follow up. This data compares favourably with the study of 25 cases by Smiley and Wasilewski (1990) [4]. They had 90\% good results at the end of 2 years and $57 \%$ of the knees continued to show improvement after 4 years. Our study has shown that arthroscopic synovectomy is useful in relieving pain, and improving motion and functional activity. This procedure removes inflamed synovium and favours the local action of drugs (biomechanical indication) [5]. Our patients had a brief hospital stay of 1.8 days on an average. There was no need for postoperative manipulation under anaesthesia in any of the patients, and no functionally significant deterioration in range of motion at the end of 3 years. The maintenance of the benefits achieved and its later deterioration are on par with all previous studies [4].

A major success of arthroscopic synovectomy is its negligible rate of complications compared to open synovectomy [5]. Our series recorded no operative or postoperative complications with regard to bleeding, wound complications, neurovascular damage, post-operative pain and fibroarthrosis. Painless effusion was seen in $20 \%$ of patients up to 3 months post-operatively, that gradually settled. Radiation synovectomy after an interval of 6-8 weeks can be a good adjunct in the future to control recurrence and to treat the residual problems following arthroscopic synovectomy [3]. The repeatability of this technique because of its mild surgical insult gives it much advantage in treating recurrent cases. 


\section{References}

1. Laurin CA, Desmarchais J, Daziano L, Gariepy R, Derome A. Long-term results of synovectomy of the knee in rheumatoid patients. Journal of Bone and Joint Surgery 1974; 56: 521-31.

2. Klug S, Wittmann G, Weseloh G. Arthroscopic synovetomy of the knee joint in early cases of rheumatoid arthritis: Follow-up results of a multicenter study. Arthroscopy 2000; 16: 262-71.

3. Ogilvie-Harris DJ, Weisleder L. Arthroscopic synovectomy of the knee: Is it helpful? Arthroscopy 1995; 11: $91-5$.

4. Smiley P, Wasilewski SA. Arthroscopic synovectomy. Arthroscopy 1990; 6: 18-23.

5. Fiocco U, Cozzi L, Rigon C, Chieco-Bianchi F, Baldovin M, et al. Arthroscopic synovectomy in rheumatoid and psoriatic knee joint synovitis: long-term outcome. British Journal of Rheumatology 1996; 35: 463-70. 